\title{
Variable angle photoelectron spectroscopy of the fluoroethylenes ${ }^{\text {a) }}$
}

\author{
Jeffrey A. Sell ${ }^{(b)}$ and Aron Kuppermann \\ Arthur Amos Noyes Laboratory of Chemical Physics, ${ }^{c)}$ California Institute of Technology, Pasadena, \\ California 91125 \\ (Received 28 August 1978)

\begin{abstract}
He I photoelectron spectra of fluoroethylene, 1,1-difluoroethylene, cis-1,2-difluoroethylene, trans-1,2difluoroethylene, trifluoroethylene, and perfluoroethylene were obtained over the scattering angle range of $45^{\circ}$ to $120^{\circ}$ and compared with those of ethylene. Vibrational frequencies of the ionic states were measured and their symmetry modes assigned. The asymmetry parameter $\boldsymbol{\beta}$ as a function of the ionization potential was measured for each molecule. The value of $\beta$ for the first ionization potential band of these molecules was found to decrease monotonically with increasing fluorine substitution. This variation was interpreted as being due to resonance mixing of the lone pair $\mathrm{F} \pi$ orbitals with $\mathrm{C}-\mathrm{C} \pi$ orbitals. The data obtained were used to assign some of the spectral bands observed.
\end{abstract}

\section{INTRODUCTION}

In the last ten years, the results of measurements of the angular distributions in the photoelectron spectroscopy of atoms and molecules have been used to characterize the symmetry, energy, and bonding nature of the orbitals from which the electrons are photoejected. ${ }^{1-9}$ They have also been used to characterize the physics of the ionization process itself. ${ }^{9(0),(t)}$ Briefly, the differential cross section $\left(d \sigma_{i f} / d \Omega\right)$ for photoionization of an atom or molecule by unpolarized light has been found to have the form ${ }^{10,11}$

$$
\frac{d \sigma_{i f}}{d \Omega}=\frac{\sigma_{i f}}{4 \pi}\left[1-\frac{\beta_{i f}}{2} P_{2}(\cos \theta)\right],
$$

where $\sigma_{i f}$ is the integral cross section for photoionization from an initial state $i$ of the atom or molecule to a final state $f$ of the corresponding singly charged ion, $P_{2}$ is the second-order Legendre polynomial, $\theta$ is the angle between the direction of the incident light beam and the momentum vector of the ejected electron, and $\beta_{i f}$ is the asymmetry parameter which ranges from -1 to +2 .

This $\beta$ parameter is the main quantity of interest in the present paper since it contains useful chemical and physical information that cannot be obtained from fixedangle spectra alone. It is now possible to perform approximate calculations of $\beta$ for atoms ${ }^{12}$ and $\mathrm{H}_{2}{ }^{13}$ which are in reasonable agreement with experiment, but for larger molecular systems, practical computational considerations require less realistic approximations to be made. This has led to results which are in substantial disagreement with experiment. ${ }^{13(2), 14}$ Although the experiments are themselves difficult to perform due to low signal intensity and perturbation by external forces such as magnetic fields, there is now fairly good agreement among the values of $\beta$ obtained by various laboratories, ${ }^{2(b),(c), 5-7,9(a),(d),(b),(t),(b)}$ and typical error bars are currently 0.1 or less.

\footnotetext{
a) This work was supported in part by a contract (EY-76-5-03767) from the U. S. Department of Energy, Report Code: CALT-767P4-177.

b) Work performed in partial fulfillment of the requirements for the Ph. D. degree in chemistry at the California Institute of Technology.

${ }^{c)}$ Contribution No. 5843 .
}

It has previously been shown that the value of $\beta$ is usually higher by at least 0.2 for ionization from $\pi$ orbitals than from $\sigma$ orbitals in molecules such as ethylene, ${ }^{2(d), 3,4(c), 9(d)}$ methyl-substituted ethylenes, ${ }^{9(a)}$ linear dienes, ${ }^{9(2)}$ and the five membered ring heterocyclic molecules furan, thiophene, and pyrrole. ${ }^{9(c)}$ We have previously used the trends in $\beta$ in going from benzene to its monohalogenated derivatives to extend some of the photoelectron band assignments of benzene to these molecules. ${ }^{9 \text { (a) }}$ It has also been found that the value of $\beta$ is roughly the same for two components of a band split by Jahn-Teller interactions. ${ }^{4(b)}$ This empirical fact has been used in cyclopropane ${ }^{8}$ and $\mathrm{SF}_{6}{ }^{9(b)}$ to assign the transitions that are split by such interactions and to rule out this possible splitting for other bands in the spectra. Finally, recent values of $\beta$ for the lowest $\pi$ band in the fluoroethylene series were compared with those obtained for some of the fluorine substituted benzenes. ${ }^{9(d)}$ It was found that $\beta$ decreases upon increasing fluorine substitution but it decreases more rapidly in the ethylene series than in the benzene series. The results were attributed

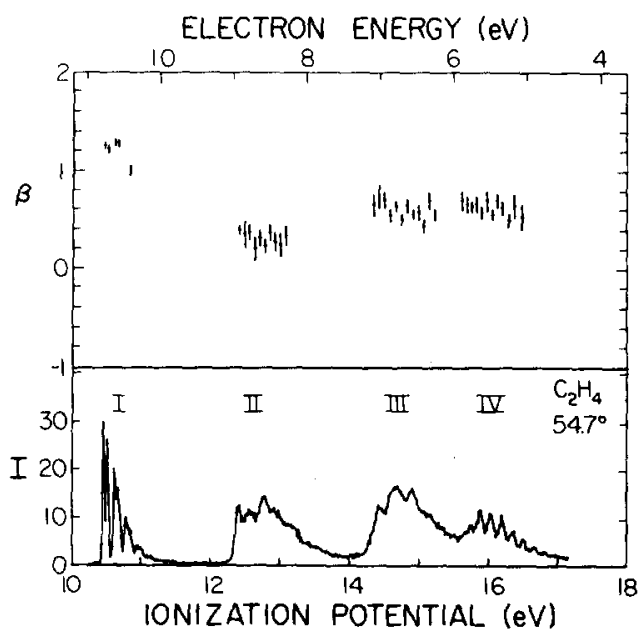

FIG. 1. Photoelectron spectrum (lower panel) and $\beta$ spectrum (upper panel) for ethylene. The former was obtained at a detector angle of $54.7^{\circ}$. This figure is reproduced from Ref. $9(\mathrm{~g})$. (The ordinate of the photoelectron spectrum of that reference was rescaled to correspond to the conditions of the other spectra in the present paper.) 
TABLE I. $\beta$ values and ionization potentials for ethylene at $584 \AA$. a

\begin{tabular}{|c|c|c|c|c|c|c|}
\hline \multirow[b]{3}{*}{ Band } & \multirow{3}{*}{$\begin{array}{l}\text { Initial } \\
\text { orbital }^{\text {b }}\end{array}$} & \multirow{3}{*}{$\begin{array}{l}\text { Orbital } \\
\text { description }\end{array}$} & \multirow{3}{*}{$\begin{array}{l}\text { Vertical } \\
\text { I. P. }(\mathrm{eV})^{\mathrm{c}}\end{array}$} & \multicolumn{3}{|c|}{$\beta$} \\
\hline & & & & \multirow[b]{2}{*}{ Range $^{d}$} & \multicolumn{2}{|c|}{ Vertical $^{\mathrm{e}}$} \\
\hline & & & & & Ref. $9(\mathrm{~g})$ & Ref. $4(\mathrm{c})$ \\
\hline $\bar{I}$ & $1 b_{3 u}$ & $\pi \mathrm{C}-\mathrm{C}$ & 10.51 & $1.0 \pm 0.10$ to $1.25 \pm 0.05$ & $1.25 \pm 0.05$ & 1.20 \\
\hline II & $1 b_{3 g}$ & $\sigma \mathrm{C}-\mathrm{H}$ & 12.82 & $0.23 \pm 0.10$ to $0.40 \pm 0.09$ & $0.30 \pm 0.05$ & 0.35 \\
\hline III & $3 a_{g}$ & $\sigma \mathrm{C}-\mathrm{C}$ & 14.69 & $0.48 \pm 0.05$ to $0.73 \pm 0.07$ & $0.60 \pm 0.10$ & 0.60 \\
\hline IV & $1 b_{2 u}$ & $\sigma \mathrm{C}-\mathrm{H}$ & 15.90 & $0.41 \pm 0.15$ to $0.75 \pm 0.08$ & $0.65 \pm 0.05$ & 0.65 \\
\hline
\end{tabular}

${ }^{a}$ All the numbers reported in this table, except those in the last column, are taken from Ref. $9(\mathrm{~g})$.

We use the standard coordinate system for which the $z$ axis lies along the $\mathrm{C}=\mathrm{C}$ bond and the $y$ axis is in the plane of the molecule [R. S. Mulliken, J. Chem. Phys. 23, 1997 (1955)].

'Measured at the band maximum and having an absolute uncertainty of $\pm 0.02 \mathrm{eV}$.

The first and second values given for each band are, respectively, the corresponding lowest and highest values of $\beta$. The variation of $\beta$ across electronic bands is discussed in Sec. III. C. The uncertainties in $\beta$ are obtained from the goodness of fit of the data to Eq. (1) (see Ref. 18).

${ }^{e}$ Obtained at the vertical I. P.

to the larger extent of transfer of fluorine $\pi$ electron density to the $\mathrm{C}-\mathrm{C} \pi$ bond in the ethylene series than in the benzene series.

The purpose of the present paper is to enhance the understanding of the electronic structure of the six fluoroethylenes using the technique of variable-angle photoelectron spectroscopy. This is the first study of this kind for these molecules. It is hoped that these data will further the knowledge of the perfluoro effect, ${ }^{15}$ which is frequently used to make photoelectron band assignments. These molecules are furthermore sufficiently small to permit a systematic study of their electronic structure by $a b$ initio calculations, ${ }^{15(a), 16,17}$ which would complement the information presented here. An additional source of interest in them is the fact that they are the molecular building blocks of many polymeric systems.

In Sec. II, we outline the experimental method. We present a general discussion of the results in Sec. III A, an analysis of vibrational structure in $\mathrm{Sec}$. III B, an

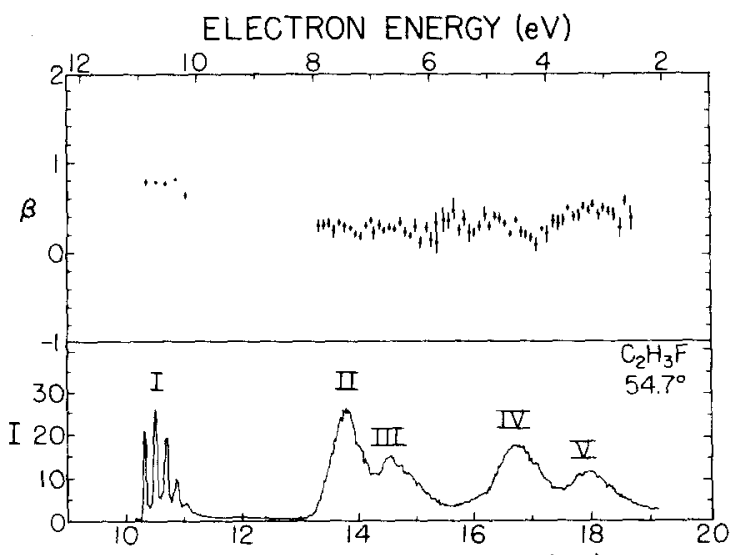

IONIZATION POTENTIAL (eV)

FIG. 2. Photoelectron spectrum (lower panel) and $\beta$ spectrum (upper panel) for fluoroethylene. The former was obtained at a detector angle of $54.7^{\circ}$ using adjacent $18 \mathrm{meV}$ wide channels and 60 scans. Total dwell time per channel was $60 \mathrm{~s}$. analysis of trends in $\beta$ in Sec. IIIC, a discussion of the initial orbital assignments in Sec. III D, and finally a summary and conclusions in Sec. IV.

\section{EXPERIMENTAL}

The apparatus used is summarized only briefly since it was described in detail previously. ${ }^{18}$ A helium discharge lamp produces He I radiation' (wavelengths of 584, 537 , and $522 \AA$ at relative intensities of $100: 2: 0,5$ ) which interacts with the sample vapor in a cylindrical scattering chamber. The pressure of the sample (typically 1 to $2 \mathrm{mTorr}$ ) is monitored by a calibrated capacitance manometer ${ }^{19}$ in the case of $\mathrm{C}_{2} \mathrm{~F}_{4}$ and by a SchulzPhelps ${ }^{20}$ ionization gauge for all other samples. The photoelectrons produced are energy dispersed by a $180^{\circ}$ hemispherical electrostatic analyzer and detected by a Spiraltron electron multiplier. The analyzer and detector are mounted on a gear wheel and can be rotated about the center of the scattering chamber, thereby scanning scattering angles from $45^{\circ}$ to $120^{\circ}$. The in-

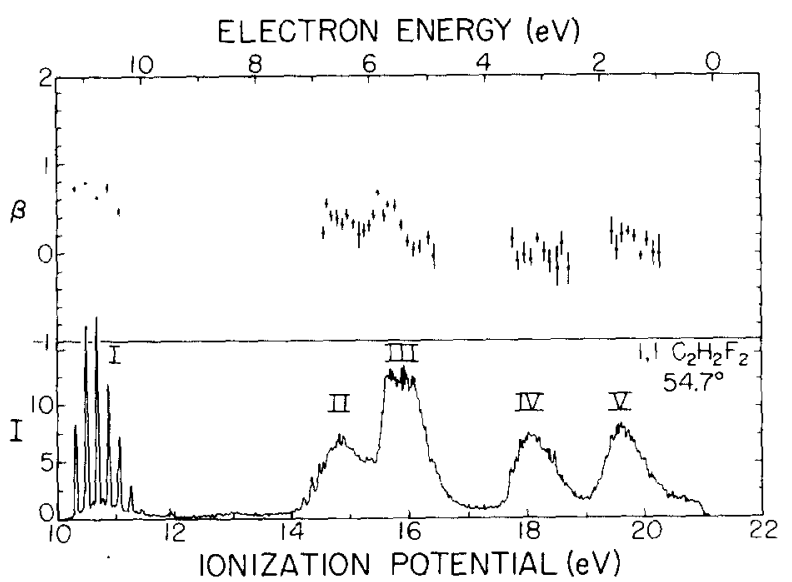

FIG. 3. Photoelectron spectrum (lower panel) and $\beta$ spectrum (upper panel) for 1,1-difluoroethylene. The former was obtained at a detector angle of $54.7^{\circ}$ using adjacent $18 \mathrm{meV}$ wide channels and 30 scans. Total dwell time per channel was $30 \mathrm{~s}$. 
TABLE II. $\beta$ values and ionization potentials for fluoroethylene at $584 \AA$.

\begin{tabular}{|c|c|c|c|c|c|c|c|c|c|c|}
\hline \multirow[b]{2}{*}{ Band } & \multicolumn{3}{|c|}{ Initial orbital } & \multirow{2}{*}{$\begin{array}{l}\text { Orbital } \\
\text { description }\end{array}$} & \multicolumn{4}{|c|}{ Vertical I. P. (eV) } & \multicolumn{2}{|l|}{$\beta^{2}$} \\
\hline & Ref. 25 & Ref. 16 & This work & & This work ${ }^{b}$ & Ref. 25 & Ref. 26 & Ref. $27^{\mathrm{c}}$ & Range & Vertical \\
\hline I & $2 a^{\prime \prime}$ & $2 a^{\prime \prime}$ & $2 a^{\prime \prime}$ & $\pi \mathrm{C}-\mathrm{C}$ & 10.56 & 10.57 & 10.58 & 10.5 & $0.67 \pm 0.05$ to $0.85 \pm 0.02$ & $0.82 \pm 0.02$ \\
\hline II & $7 a^{\prime}$ & $7 a^{\prime}$ & $\left(1 a^{\prime \prime}, 7 a^{\prime}\right)$ & $\pi n_{F}, \sigma n_{F}$ & 13.80 & 13.80 & 13.79 & 13.8 & $0.21 \pm 0.06$ to $0.33 \pm 0.05$ & $0.31 \pm 0.07$ \\
\hline III & $6 a^{\prime}$ & $6 a^{\prime}$ & $6 a^{\prime}$ & $\sigma \mathrm{C}-\mathrm{F}$ & 14.54 & 14.56 & 14.51 & 14.6 & $0.22 \pm 0.05$ to $0.36 \pm 0.11$ & $0.31 \pm 0.05$ \\
\hline IV & $5 a^{\prime}$ & $1 a^{\prime \prime}$ & $5 a^{\prime}$ & $\sigma \mathrm{C}-\mathrm{H}$ & 16.68 & 16.76 & 16.77 & 16.9 & $0.10 \pm 0.07$ to $0.40 \pm 0.10$ & $0.38 \pm 0.05$ \\
\hline $\mathrm{v}$ & $1 a^{\prime \prime}$ & $5 a^{\prime}$ & $4 a^{\prime}$ & $\sigma \mathrm{C}-\mathrm{H}$ & 18.00 & 17.97 & 17.97 & 18.2 & $0.29 \pm 0.13$ to $0.59 \pm 0.07$ & $0.54 \pm 0.06$ \\
\hline
\end{tabular}

"The meaning of the uncertainties and of "range" and "vertical" are the same as in Table I.

The absolute uncertainties are $\pm 0.02 \mathrm{eV}$.

'Values estimated from Fig. 8.33 of Ref. 27.

strument is surrounded by a $\mu$ metal shield and is located at the central region of three pairs of Helmholtz coils over which the residual magnetic field is about 0.3 $\mathrm{mG}$ or less. The background signal is subtracted from the spectra before $\beta$ is calculated. The performance of the apparatus is verified by its ability to reproduce the known value of $\beta=0.88 \pm 0.02$ for the formation of the ${ }^{2} P_{3 / 2}$ state of the argon ion by $584 \AA$ radiation. ${ }^{18}$ The energy resolution of the apparatus is measured by the full width at half-maximum of that ${ }^{2} P_{3 / 2}$ peak. The full spectra (Figs. 1 through 7) and angular distributions were obtained with the resolution set at $55 \mathrm{meV}$. Spectra of bands which have vibrational structure were also obtained at the higher resolution of $20 \mathrm{meV}$. The energy scale was calibrated by adding a small fractional amount $(<10 \%)$ of argon to the samples.

The commercial sources and stated minimum purities of the samples are as follows: $\mathrm{C}_{2} \mathrm{~F}_{4}$, Peninsular Chemical Research (PCR), 97\%; $\mathrm{C}_{2} \mathrm{HF}_{3}$, PCR, 97\%; cis-1, 2$\mathrm{C}_{2} \mathrm{H}_{2} \mathrm{~F}_{2}$, PCR, 97\%; trans -1, 2- $\mathrm{C}_{2} \mathrm{H}_{2} \mathrm{~F}_{2}$, PCR, 97\%; 1, 1 $\mathrm{C}_{2} \mathrm{H}_{2} \mathrm{~F}_{2}$, Matheson, $99 \% ; \mathrm{C}_{2} \mathrm{H}_{3} \mathrm{~F}$, Matheson, $99.9 \%$; and $\mathrm{C}_{2} \mathrm{H}_{4}$, J. T. Baker, Chemical Purity (typically $99.5 \%$ ). These samples were used without further purification.

\section{RESULTS AND DISCUSSION}

\section{A. General features of photoelectron and $\beta$ spectra}

We have obtained the photoelectron spectra and measured $\beta$ as a function of ionization potential (I. P.) and photoelectron kinetic energy (hereafter called $\beta$ spec- tra) for ethylene, fluoroethylene, 1,1-difluoroethylene, cis-1, 2-difluoroethylene, trans-1, 2-difluoroethylene, trifluoroethylene, and perfluoroethylene. The spectra shown in the bottom panels of Figs. 1 through 7 were acquired at the "magic" detector angle of $54.7^{\circ}$, for which $P_{2}$ vanishes. It can be seen from Eq. (1) that, at this angle, the ratio of the differential cross sections for two transitions is independent of their respective $\beta$ 's and is equal to the ratio of the corresponding integral cross sections. ${ }^{21,22}$

\section{1. $\mathrm{C}_{2} \mathrm{H}_{4}$}

The ethylene photoelectron and $\beta$ spectra are reproduced in Fig. 1 from Ref. 9(g) for comparison with those of its fluorine derivatives. The values of the vertical I. $P$. and of $\beta$ for ethylene obtained in previous studies $^{4(c), 9(8)}$ are listed in Table I for similar reasons. The ethylene spectrum and assignments of the bands to the corresponding orbitals are well known. ${ }^{23,24}$ The important feature of the values of $\beta$ for this molecule is that they are noticeably higher for the $\pi$ band than for the $\sigma$ bands. This behavior has been noticed not only for ethylene $e^{3,4(c), 9(d),(z)}$ but for other molecules as well. ${ }^{9(a),(c),(B),(h)}$

\section{2. $\mathrm{C}_{2} \mathrm{H}_{3} \mathrm{~F}$}

The photoelectron and $\beta$ spectra of fluoroethylene are displayed in Fig. 2. The values of the vertical I. P.'s and of $\beta$ for this molecule are listed in Table II. Previous measurements of these I. P.'s are included, ${ }^{25-27}$

TABLE III. $\beta$ values and ionization potentials for 1,1-difluoroethylene at $584 \AA$.

\begin{tabular}{|c|c|c|c|c|c|c|c|c|c|}
\hline \multicolumn{4}{|c|}{ Initial orbital } & \multirow{2}{*}{$\begin{array}{l}\text { Orbital } \\
\text { description }\end{array}$} & \multicolumn{3}{|c|}{ Vertical I.P. (eV) } & \multicolumn{2}{|l|}{$\beta^{\mathbf{a}}$} \\
\hline Band & This work & Ref. 29 & Ref. 30 & & This work ${ }^{b}$ & Ref. $28^{c}$ & Ref. 26 & Range & Vertical \\
\hline$I$ & $2 b_{1}$ & $b_{1}$ & $b_{1}$ & $\pi \mathrm{C}-\mathrm{C}$ & 10.69 & 10.84 & 10.72 & $0.46 \pm 0.05$ to $0.81 \pm 0.03$ & $0.64 \pm 0.03$ \\
\hline II & & $a_{1}, b_{2}$ & $b_{2}$ & & 14.83 & 14.85 & 14.79 & $0.19 \pm 0.17$ to $0.56 \pm 0.07$ & $0.39 \pm 0.11$ \\
\hline III & $\begin{array}{l}\text { (two } \\
\text { orbitals) }^{d}\end{array}$ & $b_{2}, a_{2}$ & $a_{1}, b_{2}, a_{2}$ & & 15.73 & 15.75 & 15.73 & $-0.05 \pm 0.17$ to $0.69 \pm 0.04$ & $0.55 \pm 0.05$ \\
\hline IV & & $a_{1}$ & & & 18.18 & 18.20 & 18. 22 & $-0.19 \pm 0.20$ to $0.15 \pm 0.13$ & $-0.07 \pm 0.11$ \\
\hline $\mathbf{v}$ & & $b_{1}$ & & & 19.74 & 19.65 & 19.68 & $-0.04 \pm 0.07$ to $0.25 \pm 0.18$ & $0.18 \pm 0.09$ \\
\hline
\end{tabular}

The meaning of the uncertainties and of range and vertical are the same as in Table 1 .

'The absolute uncertainties are $\pm 0.02 \mathrm{eV}$.

The values given are estimated from the Fig. 8.36 of Ref. 8 .

See Sec. III.D. 4. 


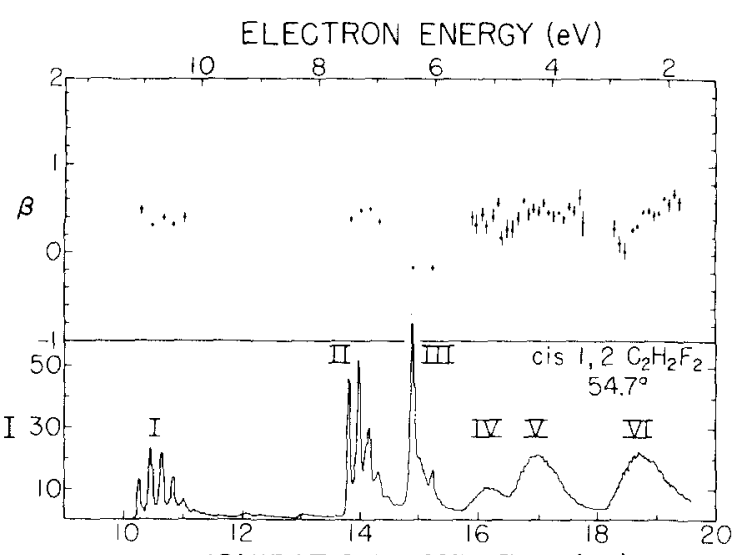

IONIZATION POTENTIAL (eV)

FIG. 4. Photoelectron spectrum (lower panel) and $\beta$ spectrum (upper panel) for cis-1,2-difluoroethylene. The former was obtained at a detector angle of $54.7^{\circ}$ using adjacent $18 \mathrm{meV}$ wide channels and 60 scans. Total dwell time per channel was $60 \mathrm{~s}$.

and show reasonable agreement with the present ones. Assignment of the initial orbitals for this and the other molecules in the series will be discussed later in this section. The only band in the spectrum of Fig. 2 showing pronounced vibrational structure is band $\mathrm{I}$, which is associated with the $\mathrm{C}-\mathrm{C} \pi$ bond (see Sec. III D). The most interesting feature of this spectrum is the lack of a sharp peak associated with ionization from a fluorine lone pair orbital. ${ }^{9(a)}$ In the $\beta$ spectrum, it can be seen that the values of $\beta$ for band I have decreased to the range 0.67 to 0.85 compared with the corresponding 1.00 to 1.25 range for ethylene. This trend towards lower $\beta$ for the $C-C \pi$ ionization continues in the rest of the molecules in this series and is discussed in detail in Sec. IIIC. Also striking is the lack of significant variation of the band average $\beta$ among bands II through $V$. The $\beta$ values for bands II and $I V$ are somewhat lower than the corresponding ones for ethylene.

\section{3. $1,1-\mathrm{C}_{2} \mathrm{H}_{2} \mathrm{~F}_{2}$}

The photoelectron and $\beta$ spectra of 1,1-difluoroethylene are given in Fig. 3. The corresponding I. P.'s and values of $\beta$ are listed in Table III, together with I. P.'s previously published. ${ }^{26,28}$ Orbital assignments previously made ${ }^{29,30}$ are also included in the table. There

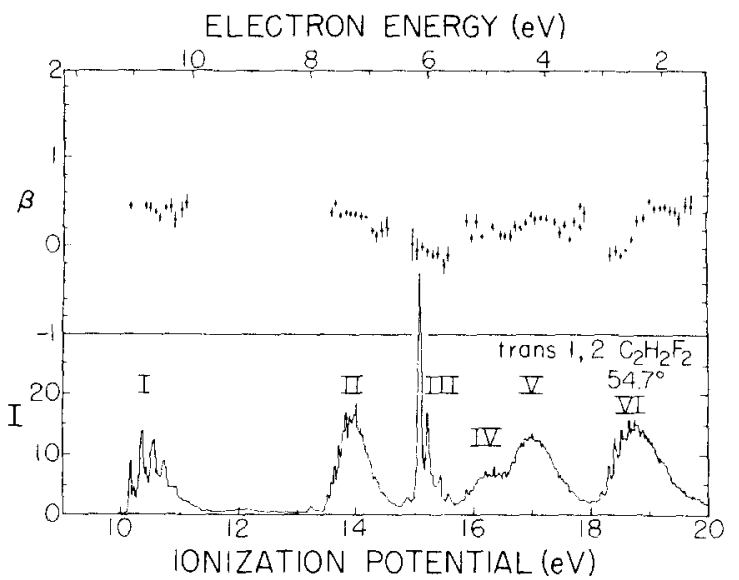

FIG. 5. Photoelectron spectrum (lower panel) and $\beta$ spectrum (upper panel) for trans-1,2-difluoroethylene. The former was obtained at a detector angle of $54.7^{\circ}$ using adjacent $18 \mathrm{meV}$ wide channels and 40 scans. Total dwell time per channel was $40 \mathrm{~s}$.

is a larger disagreement than expected among the several measurements of the vertical I. P. of band I. The photoelectron spectrum shows a significant vibrational progression in band $I$ and the values of $\beta$ for this band are lower than those of the previous molecules. There is also some resolvable structure on the rising side of band II. Band III has a relatively high intensity, and there is a substantial decrease in $\beta$ across this band. This indicates that more than one transition may be involved in this feature. The intensities and overall shapes of bands IV and V are similar to those of band II.

\section{4. cis-1,2- $\mathrm{C}_{2} \mathrm{H}_{2} \mathrm{~F}_{2}$}

The photoelectron and $\beta$ spectra of cis-1,2-difluoroethylene are displayed in Fig. 4. The values of $\beta$ and vertical I. P.'s for this molecule are listed in Table IV, together with previously published I. P. values. ${ }^{15(a)}$ Agreement with the latter is good. The interesting features in the $\beta$ spectrum are the low value of $\beta(0.30$ to 0.47 ) for band I compared with ethylene and the low value $(-0.17)$ for band III compared with the other bands

\section{5. trans-1,2- $\mathrm{C}_{2} \mathrm{H}_{2} \mathrm{~F}_{2}$}

The photoelectron and $\beta$ spectra of trans-1, 2-difluoroethylene are displayed in Fig. 5. The corresponding

TABLE IV. $\beta$ values and ionization potentials for $c i s-1,2-\mathrm{C}_{2} \mathrm{H}_{2} \mathrm{~F}_{2}$ at $584 \AA$.

\begin{tabular}{|c|c|c|c|c|c|c|}
\hline \multirow[b]{2}{*}{ Band } & \multirow{2}{*}{$\begin{array}{l}\text { Initial } \\
\text { orbital }\end{array}$} & \multirow{2}{*}{$\begin{array}{l}\text { Orbital } \\
\text { description }\end{array}$} & \multicolumn{2}{|c|}{ Vertical I. P. (eV) } & \multicolumn{2}{|l|}{$\beta^{\mathrm{a}}$} \\
\hline & & & This work ${ }^{b}$ & Ref. 15(a) & Range & Vertical \\
\hline I & $2 b_{1}$ & $\pi \mathrm{C}-\mathrm{C}$ & 10.44 & 10.43 & $0.30 \pm 0.04$ to $0.47 \pm 0.06$ & $0.30 \pm 0.03$ \\
\hline II & $\left(5 a_{1}, 4 b_{2}\right)$ & $\sigma n_{F}, \sigma n_{F}$ & 13.97 & 13.97 & $0.39 \pm 0.03$ to $0.49 \pm 0.03$ & $0.47 \pm 0.03$ \\
\hline III & $1 a_{2}$ & $\sigma n_{F}$ & 14.87 & & $-0.17 \pm 0.03$ & $-0.17 \pm 0.03$ \\
\hline IV & $\left(1 b_{1}\right)$ & $\pi n_{\mathbf{F}}$ & 16.15 & & $0.17 \pm 0.09$ to $0.57 \pm 0.07$ & $0.42 \pm 0.09$ \\
\hline $\mathrm{v}$ & $\left(3 b_{2}\right)$ & $\sigma \mathbf{C F}$ & 17.05 & & $0.33 \pm 0.17$ to $0.62 \pm 0.11$ & $0.47 \pm 0.08$ \\
\hline VI & $3 a_{1}, 4 a_{2}$ & $\sigma \mathrm{CH}, \sigma \mathrm{CF}$ & 18.79 & & $0.02 \pm 0.11$ to $0.66 \pm 0.07$ & $0.46 \pm 0.03$ \\
\hline
\end{tabular}

The meaning of the uncertainties and of range and vertical are the same as in Table $I$.

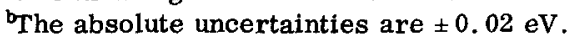


TABLE V. $\beta$ values and ionization potentials of trans-1,2-difluoroethylene at $584 \AA$.

\begin{tabular}{|c|c|c|c|c|c|c|}
\hline \multirow[b]{2}{*}{ Band } & \multirow{2}{*}{$\begin{array}{l}\text { Initial } \\
\text { orbital }\end{array}$} & \multirow{2}{*}{$\begin{array}{l}\text { Orbital } \\
\text { description }\end{array}$} & \multicolumn{2}{|c|}{ Vertical I.P. (eV) } & \multicolumn{2}{|l|}{$\beta^{2}$} \\
\hline & & & This work ${ }^{b}$ & Ref. 15(a) & Range & Vertical \\
\hline I & $2 a_{u}$ & $\pi \mathrm{C}-\mathrm{C}$ & 10.38 & 10.38 & $0.32 \pm 0.11$ to $0.53 \pm 0.11$ & $0.48 \pm 0.06$ \\
\hline II & $\left(4 b_{u}, 5 a_{g}\right)$ & $\sigma n_{F}, \sigma n_{F}$ & 13.97 & 13.90 & $0.13 \pm 0.09$ to $0.52 \pm 0.06$ & $0.39 \pm 0.05$ \\
\hline III & $1 b_{g}$ & $\sigma n_{\mathrm{F}}$ & 15.08 & & $-0.20 \pm 0.11$ to $0.04 \pm 0.20$ & $0.02 \pm 0.07$ \\
\hline IV & $\left(1 a_{u}\right)$ & $\pi n_{F}$ & 16.38 & & $0.12 \pm 0.06$ to $0.33 \pm 0.10$ & $0.21 \pm 0.04$ \\
\hline V & $\left(4 a_{g}\right)$ & $\sigma \mathrm{CF}$ & 17.00 & & $0.10 \pm 0.05$ to $0.49 \pm 0.07$ & $0.39 \pm 0.05$ \\
\hline VI & $3 a_{g}, 3 b_{u}$ & $\sigma \mathrm{CH}, \sigma \mathrm{CF}$ & 18.77 & & $-0.07 \pm 0.11$ to $0.50 \pm 0.13$ & $0.36 \pm 0.06$ \\
\hline
\end{tabular}

${ }^{2}$ The meaning of the uncertainties and of range and vertical are the same as in Table I.

${ }^{b}$ The absolute uncertainties are $\pm 0.02 \mathrm{eV}$.

values of $\beta$ and vertical I. P.'s are given in Table $V$ together with previously published ${ }^{15(\boldsymbol{k})}$ I. P.'s. The most striking aspect of the photoelectron spectrum is its similarity to that of the cis isomer (Fig. 4). The shapes and relative intensities of each of the bands is similar in the two molecules, the largest difference being the vibrational structure of bands II and VI. The corresponding vertical I.P.'s do not differ by more than $1 / 4$ $\mathrm{eV}$ in the two isomers. This is significant because the photoelectron spectra of the cis and trans isomers of compounds such as dichloro-, ${ }^{26,31}$ dibromo-, ${ }^{31,32} \mathrm{di}-$ iodo-, ${ }^{33}$ and dimethylethylene (cis- and trans-2-butene) $)^{34}$ differ from each other to a greater extent. The reason for this difference in behavior may be the small size of the fluorine atom which would lead to weaker throughspace substituent interactions in the corresponding cis isomer. [One interesting parenthetic remark is that the electronic energy of the cis-difluoroethylene is actually lower (i.e., more negative) than that of the corresponding trans isomer, ${ }^{35,36}$ contrary to a naive chemical intuition based on the assumption that the fluorine-fluorine interactions are repulsive.] The values of $\beta$ for the two isomers are generally equal to each other within experimental error, which indicates the similarity of the corresponding orbital characteristics.

\section{6. $\mathrm{C}_{2} \mathrm{HF}_{3}$}

The photoelectron and $\beta$ spectra of trifluoroethylene are given in Fig. 6. The corresponding values of $\beta$ and vertical I. P.'s are listed in Table VI together with previously published I. P. values. ${ }^{15(a)}$ The photoelectron spectrum of these molecules is more complex than those of the previous fluoroethylenes, having eight discernible bands. Again, band I has a resolvable vibrational progression, and a very low value of $\beta(0.26$ to 0.36$)$ for a $\pi$ band. There is interesting structure in the $\beta$ spectrum; in going from band I to band $V$, the value of $\beta$ decreases from 0.48 to -0.14 and then rises to a maximum of 0.51 for band VI, decreasing thereafter a minimum of -0.10 across band VIII.

\section{7. $C_{2} F_{4}$}

The photoelectron and $\beta$ spectra of tetrafluoroethylene are displayed in Fig. 7. The corresponding values of $\beta$ and vertical I.P.'s are listed in Table VII, together with previously published I. P. values. ${ }^{15(2), 26}$ The photoelectron spectrum shows an unusually large gap of almost $5.5 \mathrm{eV}$ between the vertical I. P. of bands I and II (the weak features between 14 and $15 \mathrm{eV}$ are satellites of bands II and III due to ionization by the low intensity 537

TABLE VI. $\beta$ values and ionization potentials for trifluoroethylene at $584 \AA$.

\begin{tabular}{|c|c|c|c|c|c|c|}
\hline \multirow[b]{2}{*}{ Band } & \multirow{2}{*}{$\begin{array}{l}\text { Initial } \\
\text { orbital }\end{array}$} & \multirow{2}{*}{$\begin{array}{l}\text { Orbital } \\
\text { description }\end{array}$} & \multicolumn{2}{|c|}{ Vertical I. P. (eV) } & \multicolumn{2}{|l|}{$\beta^{\mathbf{a}}$} \\
\hline & & & This work ${ }^{b}$ & Ref. 15(a) & Range & Vertical \\
\hline I & $a^{\prime \prime}$ & $\pi \mathrm{C}-\mathrm{C}$ & 10.54 & 10.53 & $0.26 \pm 0.03$ to $0.36 \pm 0.05$ & $0.30 \pm 0.03$ \\
\hline II & & & 14.62 & 14.64 & $0.16 \pm 0.12$ to $0.48 \pm 0.06$ & $0.41 \pm 0.05$ \\
\hline III & & & 15.90 & & $-0.06 \pm 0.06$ to $0.24 \pm 0.05$ & $-0.06 \pm 0.06$ \\
\hline IV & & & 16.36 & & $-0.11 \pm 0.10$ to $0.09 \pm 0.06$ & $-0.11 \pm 0.10$ \\
\hline V & & & 16.69 & & $-0.14 \pm 0.08$ to $0.04 \pm 0.11$ & $-0.08 \pm 0.03$ \\
\hline VI & & & 18.06 & & $0.25 \pm 0.07$ to $0.51 \pm 0.06$ & $0.36 \pm 0.07$ \\
\hline VII & & & 18.56 & & $0.14 \pm 0.06$ to $0.45 \pm 0.04$ & $0.18 \pm 0.06$ \\
\hline VIII & . & & 20.14 & & $-0.10 \pm 0.04$ to $0.10 \pm 0.09$ & $0.06 \pm 0.03$ \\
\hline
\end{tabular}

The meaning of the uncertainties and of range and vertical are the same as in Table I.

The absolute uncertainties are $\pm 0.02 \mathrm{eV}$. 


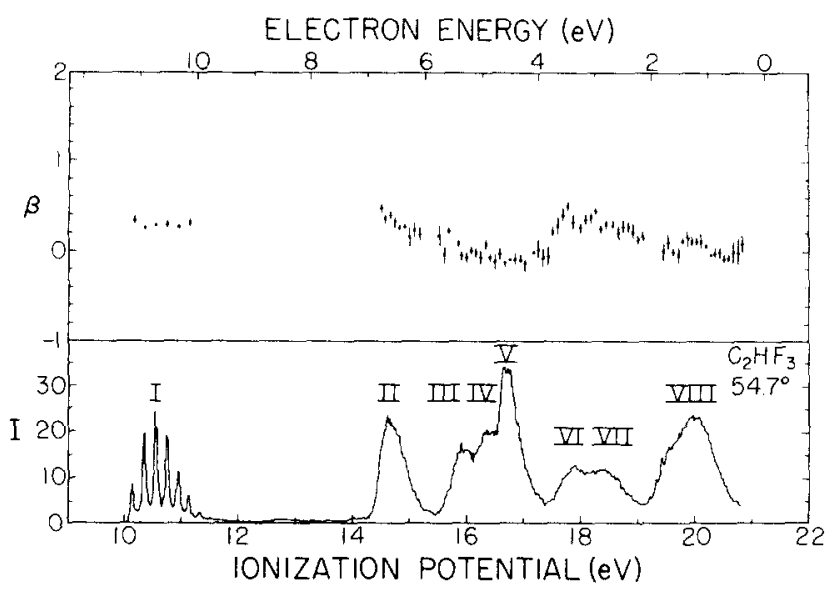

FIG. 6. Photoelectron spectrum (lower panel) and $\beta$ spectrum (upper panel) for trifluoroethylene. The former was obtained at a detector angle of $54.7^{\circ}$ using adjacent $18 \mathrm{meV}$ wide channels and 40 scans. Total dwell time per channel was $40 \mathrm{~s}$.

$\AA$ A line of our lamp). Theoretical calculations indicate the presence of at least eight transitions ${ }^{15(a), 17,37}$ from band II through $21.2 \mathrm{eV}$. The value of $\beta$ for band $\mathrm{I}$ is in the range -0.25 to 0.12 , which is very low for a $\mathrm{C}-\mathrm{C} \pi$ band. ${ }^{4(a), 9(c),(z), ~(h) ~}$

\section{B. Vibrational analysis}

High resolution spectra $(20 \mathrm{meV})$ of the bands in Figs. 1 through 7 that have resolvable structure were obtained for the purpose of making assignments of the vibrational modes. Each of these spectra has been convoluted by the five-channel smoothing procedure of Savitzky and Golay. ${ }^{38}$ In the interest of brevity, only the high resolution spectra of band I of ethylene and fluoroethylene are shown here (Figs. 8 and 9 , respectively). ${ }^{39}$ The vibrational frequencies derived from the spectra of all seven molecules are listed in Table VIII, where they are compared with frequencies previously obtained for the positive ions by photoelectron spectroscopy ${ }^{15(2), 24-28,40}$ and for the neutrals by infrared and Raman spectroscopy. ${ }^{41-45}$

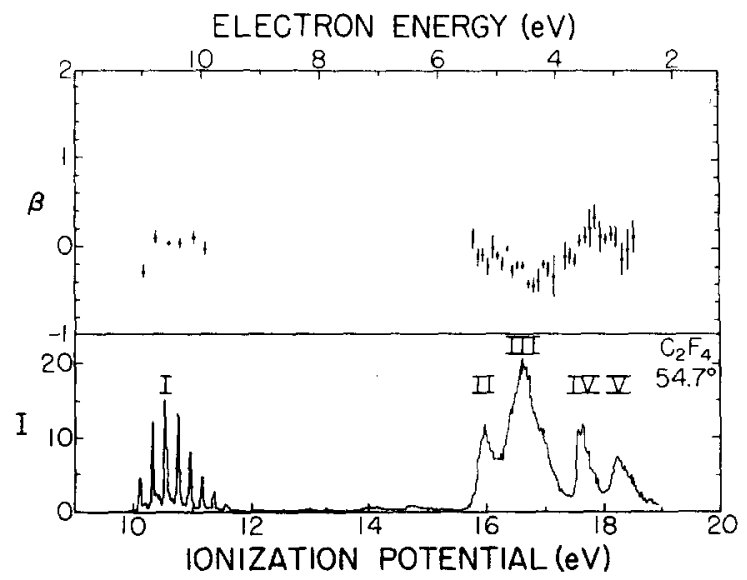

FIG. 7. Photoelectron spectrum (lower panel) and $\beta$ spectrum (upper panel) for perfluoroethylene. The former was obtained at a detector angle of $54.7^{\circ}$ using adjacent $18 \mathrm{meV}$ wide channels and 50 scans. Total dwell time per channel was $50 \mathrm{~s}$.

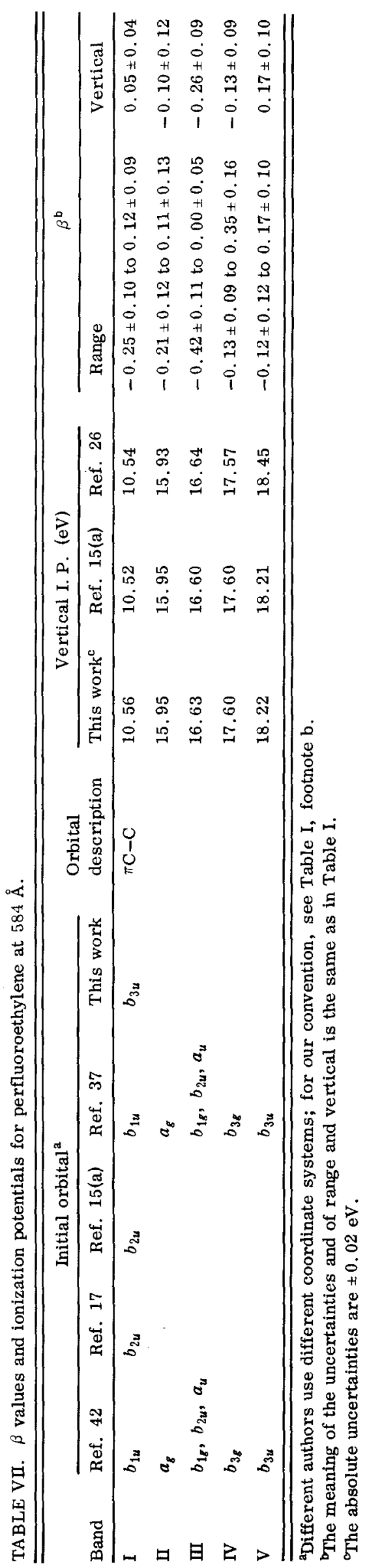

J. Chem. Phys., Vol. 71, No. 11, 1 December 1979 


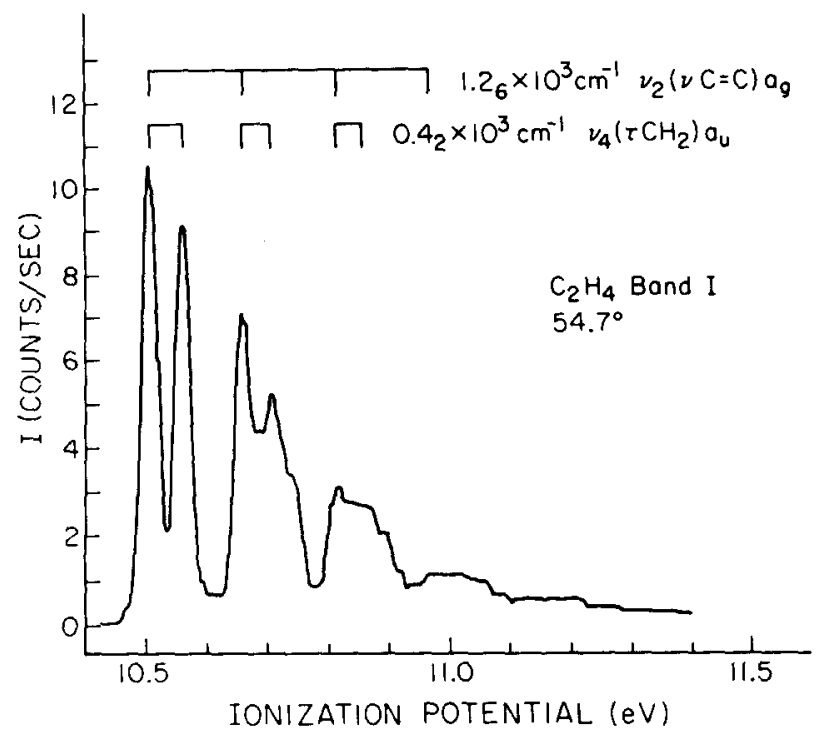

FIG. 8. High resolution $(20 \mathrm{meV})$ photoelectron spectrum of band I of ethylene obtained at a detector angle of $54.7^{\circ}$ using adjacent $3 \mathrm{meV}$ wide channels and 80 scans. Total dwell time per channel was $80 \mathrm{~s}$. The position of the vibrational lines, the assignment of the corresponding vibrational modes, and the values of their frequencies are given at the top of the figure.

Assignments of the vibrational bands of the ions are also listed in Table VIII. These assignments are made by comparison with corresponding neutral molecule frequencies and consideration of the nature of the electron removed (bonding, nonbonding, or antibonding) in going from the neutral molecule to the ion. The assignments with a greater degree of uncertainty (involving particularly large differences between the frequencies of the ion and the neutral molecule or closeness to two different frequencies of the neutral molecule) are depicted between square brackets in Table VIII.

The spectra of band I in all of these molecules consists of a main progression associated with excitation of the $\mathrm{C}=\mathrm{C}$ stretching vibration plus combination bands for which one or more additional modes are excited. In the ethylene case (Fig. 8), the additional mode is a $\nu_{4}\left(\tau \mathrm{CH}_{2}\right) a_{u}$ twisting torsion; possibly one more mode is involved. ${ }^{23}$ That the torsional mode is excited is reasonable since, in the ground electronic state of $\mathrm{C}_{2} \mathrm{H}_{4}^{+}$, the two methylene planes are twisted by $27^{\circ}$ with $\mathrm{re}$ spect to each other. ${ }^{15(\mathrm{a}), 24,40,45}$ The decrease in $\nu_{2}(\nu \mathrm{C}=\mathrm{C}) a_{\mathrm{g}}$ in going from the neutral to the ion shows that band I corresponds to ionization from a strongly bonding orbital, which, according to theoretical calculations, ${ }^{15(a), 16}$ should be the $b_{34}$ one. Our vibrational frequencies agree well with those published earlier ${ }^{24}$ for ethylene. In band I of fluoroethylene (Fig. 9), several modes are excited in addition to the main $\nu_{4}(\nu \mathrm{C}=\mathrm{C}) a_{8}$ progression. Our frequencies agree well with the previously published ${ }^{25,26}$ ones. In addition, we see a progression with a frequency spacing of $1.4_{2} \times 10^{3} \mathrm{~cm}^{-1}$, which had not been noticed before. The fact that the CF stretching frequency in band $I$ of $\mathrm{C}_{2} \mathrm{H}_{3} \mathrm{~F}$ increases from $1.1_{5} \times 10^{3} \mathrm{~cm}^{-1}$ in the neutral molecule to $1.2_{7}$ $\times 10^{3} \mathrm{~cm}^{-1}$ in the ion (see Table VIII) indicates that some
CF antibonding character is mixed into the ground state orbital.

As seen from Table VIII, the agreement between our vibrational frequencies for $1,1-\mathrm{C}_{2} \mathrm{H}_{2} \mathrm{~F}_{2}$ and those previously published ${ }^{26}$ is good, with the exception of the $1.0_{5} \times 10^{3} \mathrm{~cm}^{-1}$ mode in band II. The spectrum of cis$1,2-\mathrm{C}_{2} \mathrm{H}_{2} \mathrm{~F}_{2}$ (Fig. 4) is particularly rich in vibrational structure: at least two modes contribute to each of band I, II, and III. One interesting aspect of this molecule is that the $\nu_{2}(\nu \mathrm{C}=\mathrm{C}) a_{1}$ vibrational mode is observed for two different electronic states of the ion. As expected, the value of its frequency is different for these two states (1. $5_{7} \times 10^{3}$ and $1.4_{2} \times 10^{3} \mathrm{~cm}^{-1}$ for bands I and II, respectively). This is due to the different bonding character istics of the electron which is removed in producing the corresponding states of the ion. The spectrum of trans$1,2-\mathrm{C}_{2} \mathrm{H}_{2} \mathrm{~F}_{2}$ is also rich in vibrational structure (Fig. 5). Band $\mathrm{I}$ is rather complex, consisting of a main progression associated with excitation of the $\nu_{2}(\nu \mathrm{C}=\mathrm{C}) a_{\mathrm{g}}$ stretching vibration $\left(1.6_{0} \times 10^{3} \mathrm{~cm}^{-1}\right)$ plus combination bands associated with the $\nu_{3}(\delta \mathrm{CHF}) a_{\mathrm{g}}\left(1.2_{3} \times 10^{3} \mathrm{~cm}^{-1}\right), \nu_{4}(\nu \mathrm{CF}) a_{\mathrm{g}}$ $\left(0.8_{5} \times 10^{3} \mathrm{~cm}^{-1}\right)$, and $\nu_{5}(\delta \mathrm{CHF}) a_{\mathrm{g}}\left(0.5_{5} \times 10^{3} \mathrm{~cm}^{-1}\right)$ modes. This molecule also contains the lowest $\left(0.1_{5} \times 10^{3} \mathrm{~cm}^{-1}\right)$ and highest $\left(2.8_{2} \times 10^{3} \mathrm{~cm}^{-1}\right)$ frequencies observed in this series of seven molecules.

In the spectra of $\mathrm{C}_{2} \mathrm{~F}_{3} \mathrm{H}$ and $\mathrm{C}_{2} \mathrm{~F}_{4}$, only band $\mathrm{I}$ has vibrational structure (Figs. 6 and 7). Two vibrational frequencies are seen in $\mathrm{C}_{2} \mathrm{~F}_{3} \mathrm{H}$, one of which corresponds to the $\nu_{2}(\nu \mathrm{C}=\mathrm{C}) a^{\prime}$ stretching mode. In $\mathrm{C}_{2} \mathrm{~F}_{4}$, three modes are observed. The $\nu_{2}$ frequency is relatively high $\left(1.7_{1} \times 10^{3} \mathrm{~cm}^{-1}\right)$ and the $\nu_{1}(\nu \mathrm{CF}) a_{g}$ frequency is higher

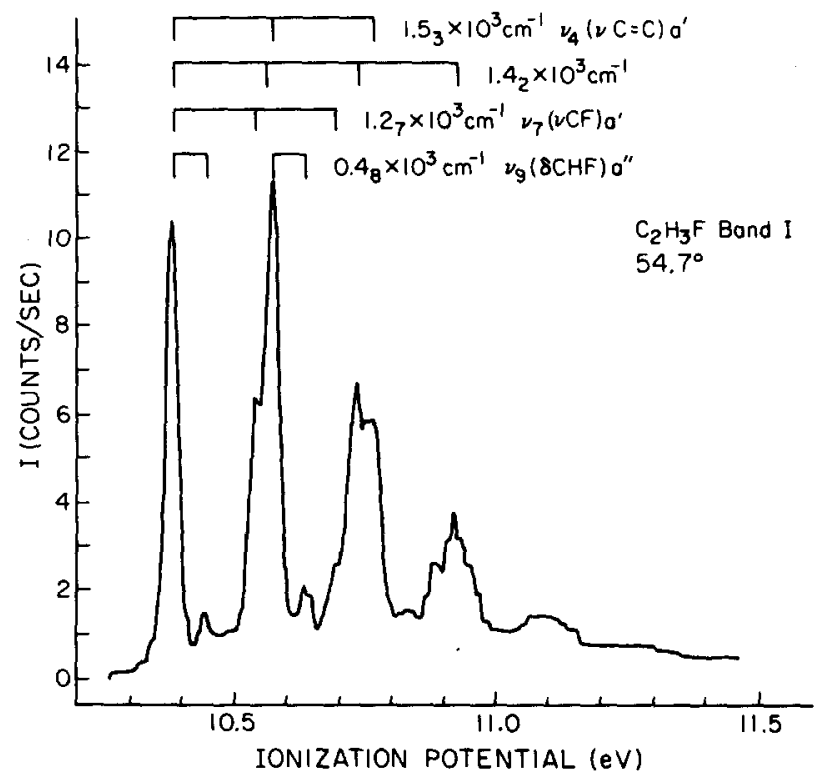

FIG. 9. High resolution $(20 \mathrm{meV})$ photoelectron spectrum of band I of fluoroethylene obtained at a detector angle of $54.7^{\circ}$ using adjacent $3 \mathrm{meV}$ wide channels and 100 scans. Total dwell time per channel was $100 \mathrm{~s}$. The position of the vibrational lines, the assignment of the corresponding vibrational modes, and the values of their frequencies are given at the top of the figure. 
TABLE VUI. Vibrational frequencies.

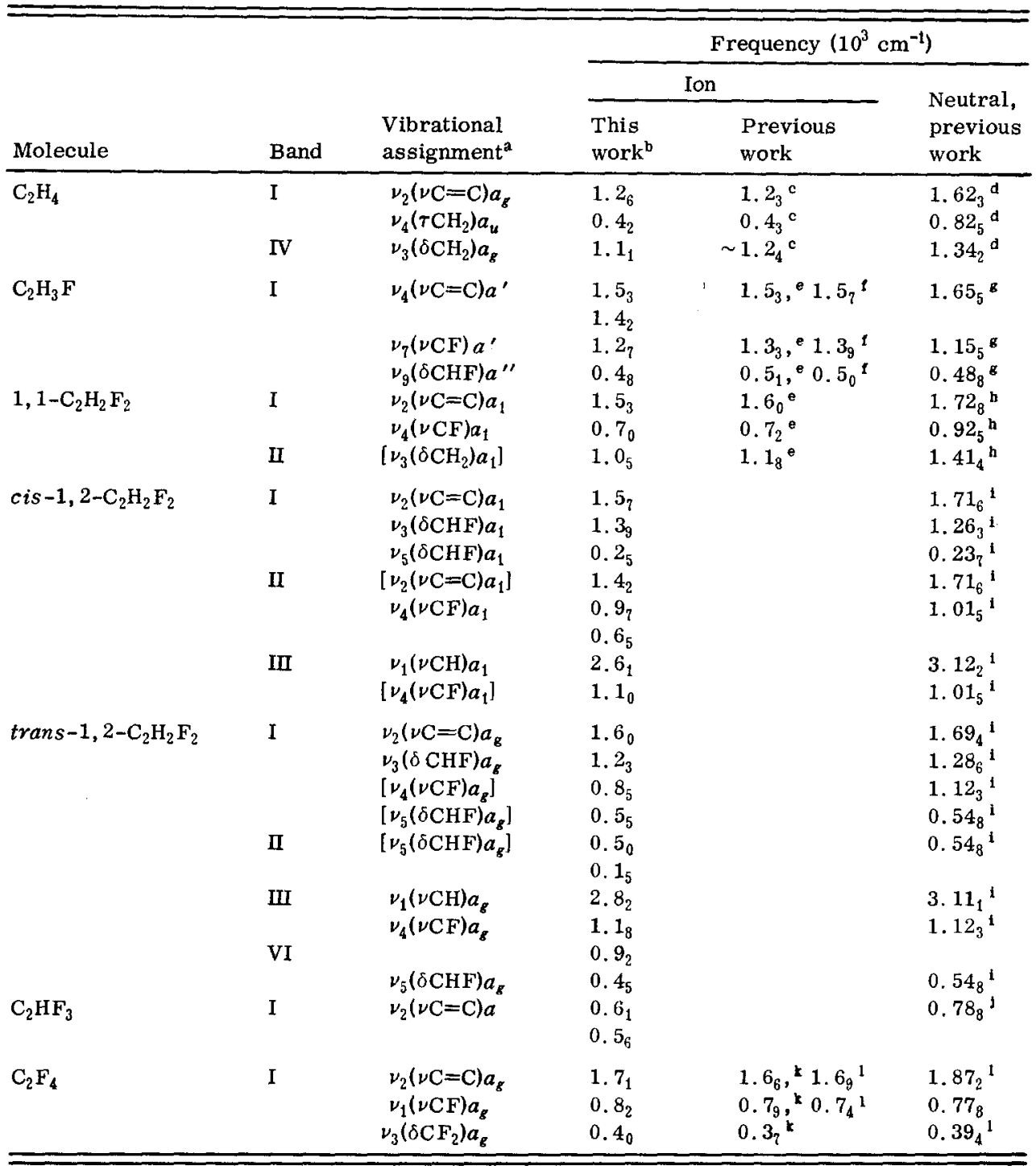

aThe notation follows the recommendation given by R. S. Mulliken, J. Chem. Phys. 23, 1997 (1955).

The absolute uncertainties are estimated to be $\pm 0.08 \times 10^{3} \mathrm{~cm}^{-1}$.

'References 23 and 24, and C. R. Brundle and D. B. Brown, Spectrochim. Acta Part A 27, 2491 (1971).

Values given in Refs. 24 and 25(a) without reference to the original work.

Reference 26.

${ }^{\text {i Reference } 25 .}$

Reference 41.

'Reference 42.

${ }^{1}$ Reference 43.

${ }^{\prime}$ Reference 44.

Reference 15(a).

${ }^{1}$ Values given in Ref. 15(a) without reference to the original work.

in the ion than in the neutral molecule, in agreement with an antibonding assignment of the orbital from which the electron was removed. ${ }^{15(2)}$ The lack of an observation of the torsional mode may indicate that the ground state of $\mathrm{C}_{2} \mathrm{~F}_{4}^{+}$is planar, ${ }^{15(2)}$ unlike the ethylene ion. Also, since the band maximum occurs for $v^{\prime}=2$ in band I of perfluoroethylene (Fig. 7), there must be a larger increase in the $\mathrm{C}-\mathrm{C}$ bond distance in going from the neutral molecule to the ion in $\mathrm{C}_{2} \mathrm{~F}_{4}$ than in $\mathrm{C}_{2} \mathrm{H}_{4} \cdot{ }^{15(\mathrm{a})}$

\section{Analysis of trends in $\beta$ spectra}

\section{First $\pi$ band}

A cursory observation of Figs. 1 through 7 indicates that the value of $\beta$ for the lowest I. P. $\pi$ band of the series of ethylenic molecules being considered depends rather significantly on the degree of fluorine substitution. Let us examine this phenomenon in greater detail. To this effect, we display in Fig. 10 the value of $\beta$ for 


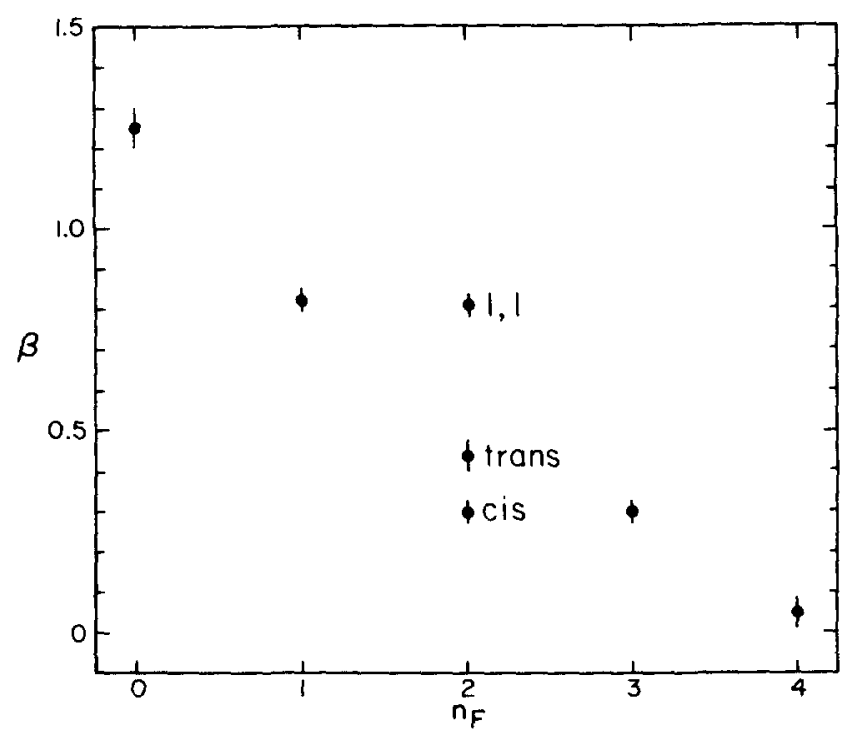

FIG. 10. Plot of $\beta$ for the $\mathrm{C}-\mathrm{C} \pi$ orbitals as a function of the number of fluorine atoms $n_{F}$ in the fluoroethylene series. The values of $\beta$ in this figure were obtained at an electron kinetic energy of $10.71 \mathrm{eV}$ (see text).

this band as a function of the number $n_{F}$ of fluorine substituents. In order to eliminate the effect on $\beta$ of photoelectron energy, we used the value of $\beta$ corresponding to the same photoelectron energy for all of these molecules, namely, $10.71 \mathrm{eV}$. This is the energy with which the "vertical" photoelectron of ethylene is ejected. The $\beta$ values in Fig. 10 were obtained by appropriately interpolating the values of Figs. 1 through 7 . The extent of this interpolation is rather small, since the vertical I. P.'s of these malecules are all within $0.2 \mathrm{eV}$ of the value for ethylene. In the absence of autoionization, this procedure is expected to be almost equivalent to the measurement of the vertical $\beta$ 's using a variable wavelength excitation source tuned to produce photoelectrons of the same kinetic energy for each molecule. ${ }^{9(f)}$

It can be seen from Fig. 10 that $\beta$ decreases monotonically with $n_{F}$ from 1.25 in ethylene to 0.05 in perfluoroethylene. This decrease is interpreted ${ }^{9(d)}$ as due to the resonance mixing ${ }^{46}$ of the fluorine lone-pair $\pi$ electrons with the $\mathrm{C}-\mathrm{C} \pi$ electrons. A quantitative analysis of this effect is not presently feasible due to the difficulty in performing accurate ab initio calculations of $\beta$ for polyatomic molecular systems and the consequent lack of detailed theoretical understanding of factors affecting molecular photoelectron angular distributions. We nevertheless suggest a qualitative explanation of this behavior of $\beta$.

The resonant mixing of the $\pi$ fluorine lone pair electrons and the $\pi \mathrm{C}-\mathrm{C}$ electrons can be crudely described by writing the resulting hybrid orbital $\psi$ as a linear combination of the fluorine lone-pair orbitals $\phi_{1}$ and the $\mathrm{C}-\mathrm{C} \pi$ orbital $\phi_{2}$ :

$$
\psi=C_{1} \phi_{1}+C_{2} \phi_{2} \text {. }
$$

Within the single configuration LCAO-MO approximation, the angular dependence of the photoelectron differential cross section comes from the square of the absolute value of the matrix element $\left\langle\psi_{e}|\hat{\epsilon} \cdot \mathbf{r}| C_{1} \phi_{1}+C_{2} \phi_{2}\right\rangle$, where $\psi_{e}$ is the wave function of the ejected photoelectron, $\hat{\boldsymbol{\epsilon}}$ is the unit vector in the polarization direction of the light beam, and $\mathbf{r}$ is the position vector of the electron. ${ }^{9(a), 14(b)}$ Multiplying this transition amplitude by its complex conjugate leads to four terms, two of which are interference cross terms which we ignore in this simplified analysis. Of the other two terms, one contains the angular dependence parameter $\beta_{1}$ of the photoelectrons ejected from the $\phi_{1}$ orbital and the other contains $\beta_{2}$ which is associated with photoionization from the $\phi_{2}$ orbital. The resulting value of $\beta$ from this hybrid orbital $\psi$ will, as a result, be a linear combination of these two $\beta^{\text {s }} \mathrm{s}$ :

$$
\beta=\frac{\left|C_{1}\right|^{2} \sigma_{1} \beta_{1}+\left|C_{2}\right|^{2} \sigma_{2} \beta_{2}}{\left|C_{1}\right|^{2} \sigma_{1}+\left|C_{2}\right|^{2} \sigma_{2}},
$$

where $\sigma_{1}$ and $\sigma_{2}$ are the corresponding integral photoionization cross sections corresponding to the orbitals $\phi_{1}$ and $\phi_{2}$. We take for $\beta_{2}$ the value for the vertical $\mathrm{C}-\mathrm{C} \pi$ band in ethylene which is 1.25 . The value of $\beta_{1}$ for the atomic fluorine lone-pair orbital has not been measured or calculated accurately. We approximate it by the value for the $\pi$ fluorine lone-pair orbital ionization in fluorobenzene which is $-0.52 .^{9(a)}$ Since, in Eq. (3), the quantities $\sigma_{1},\left|C_{1}\right|^{2}, \sigma_{2}$, and $\left|C_{2}\right|^{2}$ are all positive, the resulting $\beta$ will be lower than that for ethylene. This prediction is a consequence of the neglect of the interference terms mentioned above, and it is in agreement with our observations for all of the fluoroethylenes. It seems physically reasonable that increasing the degree of fluorine substitution would increase the fluorine character of the hybrid orbital and lower the value of $\beta$ (as long as interference terms are unimportant), as Eq. (2) predicts and as observed in Fig. 10.

We have also measured $\beta$ for the highest $\pi$ bands in some of the fluorobenzenes and found a decrease in $\beta$ upon increased fluorine substitution, but the decrease was less pronounced than in the ethylene series. ${ }^{9(d)}$ This is probably due to a smaller extent of resonance mixing in the benzene series. ${ }^{47}$

If Fig. 10 could be reproduced theoretically, more detailed information could be obtained about the bonding in these molecules. Theoretical descriptions should also strive to account for the differences in $\beta$ among the geometrical isomers of difluoroethylene. They may be due to the differences in the fluorine $\pi$ electron donation to the $\mathrm{C}=\mathrm{C}$ bond, depending on whether one or two fluorine atoms are bonded to the same carbon atom, and to the presence of fluorine-fluorine interactions. Another possible explanation is the occurrence of autoionization in these molecules. One indication of autoionization is rapid and/or nonmonotonic variation of $\beta$ with vibrational quantum number across a photoelectron. band. ${ }^{9(0),(f)}$ There is some indication of this as can be seen for band $I$ in Figs. 3 and 5. This should be further investigated by examining the photoelectron angular distribution using a synchrotron radiation light source.

\section{Higher bands}

The trends in the $\beta$ spectra for bands above $12 \mathrm{eV}$ in this series are more difficult to follow than the trends 


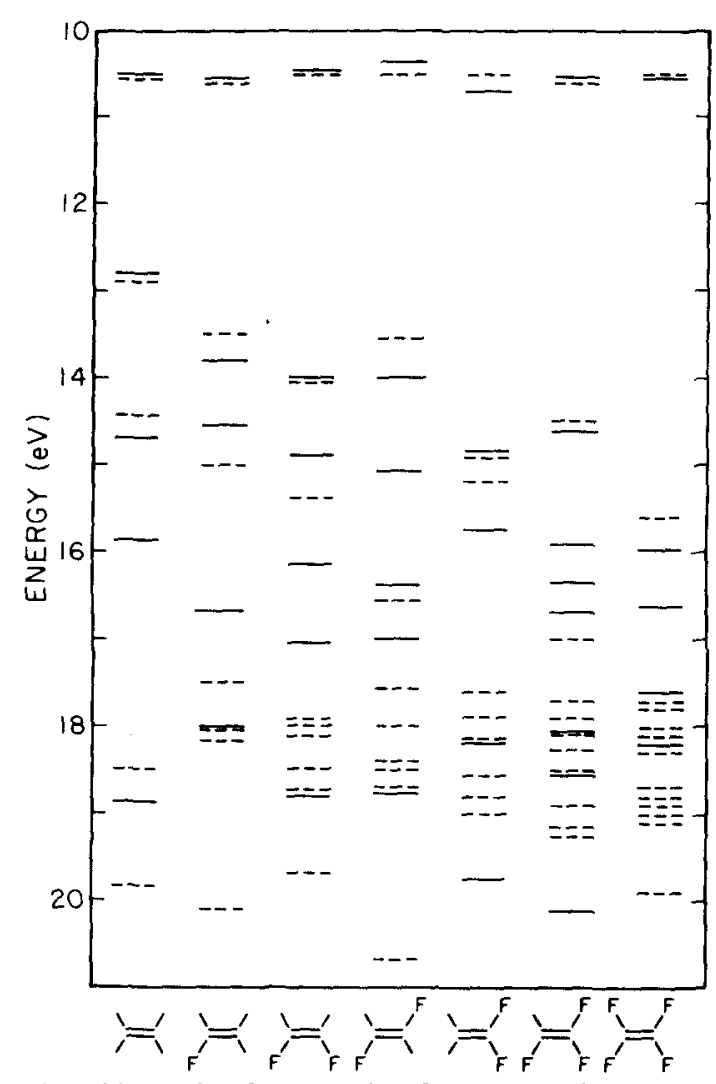

FIG. 11. Orbital energy level diagrams for the fluoroethylene series derived by photoelectron spectroscopy in the present work (solid lines) and by CNDO/2 calculations (dotted lines) from Ref. 48.

for band $I$ discussed above. The values of $\beta$ for bands II through $\mathrm{V}$ in $\mathrm{C}_{2} \mathrm{H}_{3} \mathrm{~F}$ are lower than the values for bands II through IV in $\mathrm{C}_{2} \mathrm{H}_{4}$. This decrease may be related to the mixing of some fluorine $\sigma$ electron density into the $\mathrm{C}-\mathrm{C}$ and $\mathrm{C}-\mathrm{H} \sigma$ bonds. In $1,1-\mathrm{C}_{2} \mathrm{H}_{2} \mathrm{~F}_{2}$, the decrease is even more pronounced for bands IV and $\mathrm{V}$ than in $\mathrm{CH}_{3} \mathrm{~F}$. The sharp decrease in $\beta$ from 0.69 to -0.05 across band III (Fig. 3) may indicate the presence of two transitions. In cis- (Fig. 4) and trans-1, 2- $\mathrm{C}_{2} \mathrm{H}_{2} \mathrm{~F}_{2}$ (Fig. 5), band III is particularly sharp and has a low value of $\beta$, roughly -0.2 . In analogy with band IV of fluorobenzene, ${ }^{9(\Omega)}$ these transitions could correspond to ionization mainly from the fluorine lone-pair orbitals. Also, the variation of $\beta$ across band VI in these two molecules may indicate the presence of two transitions. The low value $(-0.14)$ of $\beta$ in $\mathrm{C}_{2} \mathrm{HF}_{3}$ (Fig. 6) for the moderately sharp band $V$ may indicate some fluorine lone-pair character in the corresponding orbital. Finally, for $\mathrm{C}_{2} \mathrm{~F}_{4}$, the interesting aspect of $\beta$ is its low value for band III, which might be indicative of fluorine lone-pair ionization.

\section{Initial orbital assignments}

Hayashi and Nakajima ${ }^{48}$ have recently published the results of semiempirical $\mathrm{CNDO} / 2$ calculations on the fluoroethylenes. In Fig. 11, we compare the energy level diagrams for this series of molecules based on their results (dotted lines) and our measured ionization potentials (solid lines). This type of calculation is used $^{37}$ to make orbital assignments in photoelectron spectra, with the assumption that Koopmans' theorem ${ }^{49}$ is valid. The parameters associated with the fluorine atom which are needed to perform these semiempirical calculations were selected ${ }^{48}$ to reproduce a wide range of observed ionization potentials for mono-, 1,1-di-, and perfluoroethylene. Therefore, an assessment of the reliability of these calculations should be based mainly on the remaining molecules. From Fig. 11, it can be seen that the correlation between the calculations and measurements is good only for the highest two orbital energies of each molecule. An analysis of that figure indicates that this type of calculation is very unreliable for making orbital assignments for bands beyond the two lowest I. P. photoelectron bands. A further indication of this lack of reliability is obtained by comparing the results for cis-and trans-1,2-difluoroethylene. The theoretical energies of the bands in these two isomers are quite different, whereas the corresponding experimental values differ by less than $1 / 4 \mathrm{eV}$ from each other for the whole set of measured ionization potentials. Therefore, none of the assignments in the present paper is based upon the results of those CNDO/ 2 calculations.

1. $\mathrm{C}_{2} \mathrm{H}_{4}$

As mentioned previously, the assignments for ethylene are well known and are listed in Table I.

2. $\mathrm{C}_{2} \mathrm{H}_{3} \mathrm{~F}$

Band I has previously been assigned ${ }^{25}$ to the $2 a^{\prime \prime}$ lowest I. P. $\pi$ orbital on the basis of its vibrational structure and on the $a b$ initio calculations of Meza and Wahlgren. ${ }^{16}$ The high values of $\beta$ which we obtained for this band are consistent with this assignment. Bands II through $\mathrm{V}$ are more difficult to assign due to the lack of vibrational structure and lack of distinctive characteristics in the variation of $\beta$ across these different bands. One method of making these assignments is to compare the spectrum of fluoroethylene with those of chloro-, ${ }^{31}$ bromo-, ${ }^{32}$ and iodoethylene ${ }^{33}$ obtained previously. Figure 12 shows the vertical I. P.'s of these molecules arranged as an orbital energy level diagram. For I. P.'s below $18 \mathrm{eV}$, there are six bands each for chloro-, bromo-, and iodoethylene and five for fluoroethylene.

Correlation (dashed) lines are drawn between the four molecules; the correlations between chloro-, bromo-, and iodoethylene are straightforward, but those between chloro- and fluoroethylene are less clear. It seems reasonable that the $4 a^{\prime}, 5 a^{\prime}$, and $6 a^{\prime}$ orbitals of fluoroethylene would correlate with the corresponding ones in the other monohaloethylenes as shown in that figure, since these are $\sigma$ orbitals. Fluorine tends to shift $\sigma$ orbitals to a higher I. P. than the other halogens due to its greater inductive (electron withdrawal) effect. ${ }^{50}$ The correlation between the fluoroethylene $1 a^{\prime \prime}$ and $7 a^{\prime}$ orbitals and the corresponding ones in the other monohaloethylenes is less certain due to the lack of a peak in the fluoroethylene spectrum near $12.4 \mathrm{eV}$, which would correspond to the $7 a^{\prime}$ orbital. A peak in this region might be expected on the basis of a smooth extrapolation of the corresponding $\mathrm{I}, \mathrm{Br}$, and $\mathrm{Cl}$ levels in Fig. 12. In the absence of such a peak, one possibility is to assign the $1 a^{\prime \prime}$ and $7 d^{\prime}$ orbitals to the $13.80 \mathrm{eV}$ band. Another is to assign the $7 a^{\prime}$ orbital to the $13.80 \mathrm{eV}$ band and the 


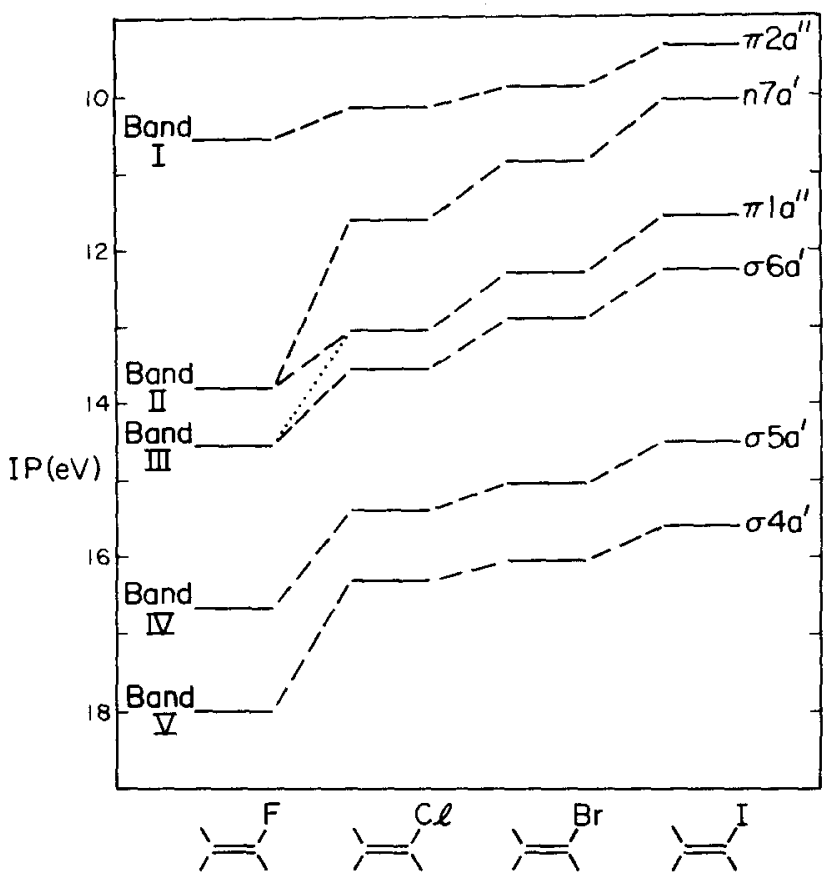

FIG. 12. Correlation of energy levels for the monohalosubstituted ethylenes derived by photoelectron spectroscopy. The orbital energies and assignments for iodoethylene are taken from Ref. 33 and for bromo- and chloroethylene from Ref. 31 . The orbital energies of fluoroethylene are those of the present experiments; the assignments for it are derived on the basis of these correlations, the $\beta$ values, and vibrational structure in the spectra (see text).

$1 a^{\prime \prime}$ one to band III at $14.54 \mathrm{eV}$, together with the $6 a^{\prime}$ orbital (as indicated by the dotted line on that figure). Unfortunately, the mild structure in the $\beta$ spectrum in the region of bands II and III does not help us to distinguish between these alternatives. In addition, since the $7 a^{\prime}$ orbital is a fluorine nonbonding one, we would expect to see a sharp intense band, which is not observed for bands II or III. Finally, adding to the confusion are the two previous conflicting assignments of the $1 a^{\prime \prime}$ orbital to band $I V^{16}$ and band V. ${ }^{25}$

These assignments seem unreasonable to us based on the above correlations with the other monohaloethylenes and the assignment of the equivalent orbital in fluorobenzene to a band at $13.89 \mathrm{eV} .^{9(a)}$ The only argument for an assignment of the $1 a^{\prime \prime}$ orbital to band II (13.80 $\mathrm{eV}$ ) rather than band III $(14.54 \mathrm{eV})$ is that the intensity of band II is larger than the other bands in the spectrum, as could be expected if both the $7 a^{\prime}$ and $1 a^{\prime \prime}$ ionizations were contributing to it. This point could be clarified by performing accurate $a b$ initio calculations of the corresponding states of the positive fluoroethylene ion, as well as of the ground state of the neutral molecule.

\section{3. cis- and trans-1,2- $\mathrm{C}_{2} \mathrm{H}_{2} \mathrm{~F}_{2}$}

The orbital assignments for these molecules are assumed to be the same since, as discussed in Sec. III, the spectra are so similar to each other. There is no question that band I corresponds to the highest occupied $\pi$ orbital based on its low I. P. and the large separation of its vibrational components which yield a vibration fre- quency characteristic of $\mathrm{C}=\mathrm{C}$ bonds. This orbital would be the $2 a_{u}$ in the trans isomer and the $2 b_{1}$ in the cis isomer. The low value of $\beta$ for band III indicates that it is a nonbonding fluorine orbital, of unknown symmetry, in analogy with the nonbonding orbitals in fluorobenzene. ${ }^{g(a)}$ It is somewhat surprising that this band shows vibrational structure. For a pure lone-pair ionization, one would expect such structure to be absent and the band to consist of a single, very sharp peak, as in chloro-, bromo-, and iodobenzene. ${ }^{9(2)}$ Since, as indicated in Table VIII, both $\mathrm{C}-\mathrm{F}$ and $\mathrm{C}-\mathrm{H}$ vibrations contribute to that band, one concludes that the orbital involved has not only some $\mathrm{C}-\mathrm{F}$ character but, more surprisingly, some $\mathrm{C}-\mathrm{H}$ character also.

The correlations between the energy levels of the trans-dihaloethylenes can be of similar usefulness as those between the vinyl compounds. These correlations between trans-1, 2-di-iodo-, -dibromo-, dichloro-, and -difluoroethylene are shown in Fig. 13. Also indicated on that figure are assignments based on those correlations and on the arguments presented below. The orbital designations are those for the trans isomer symmetry. The correlation of the $4 b_{u}$ and $5 a_{g}$ fluorine nonbonding orbitals with the corresponding ones in the other dihalo compounds seems reasonable, based on the trend toward higher I. P. as one goes from di-iodo- to di-

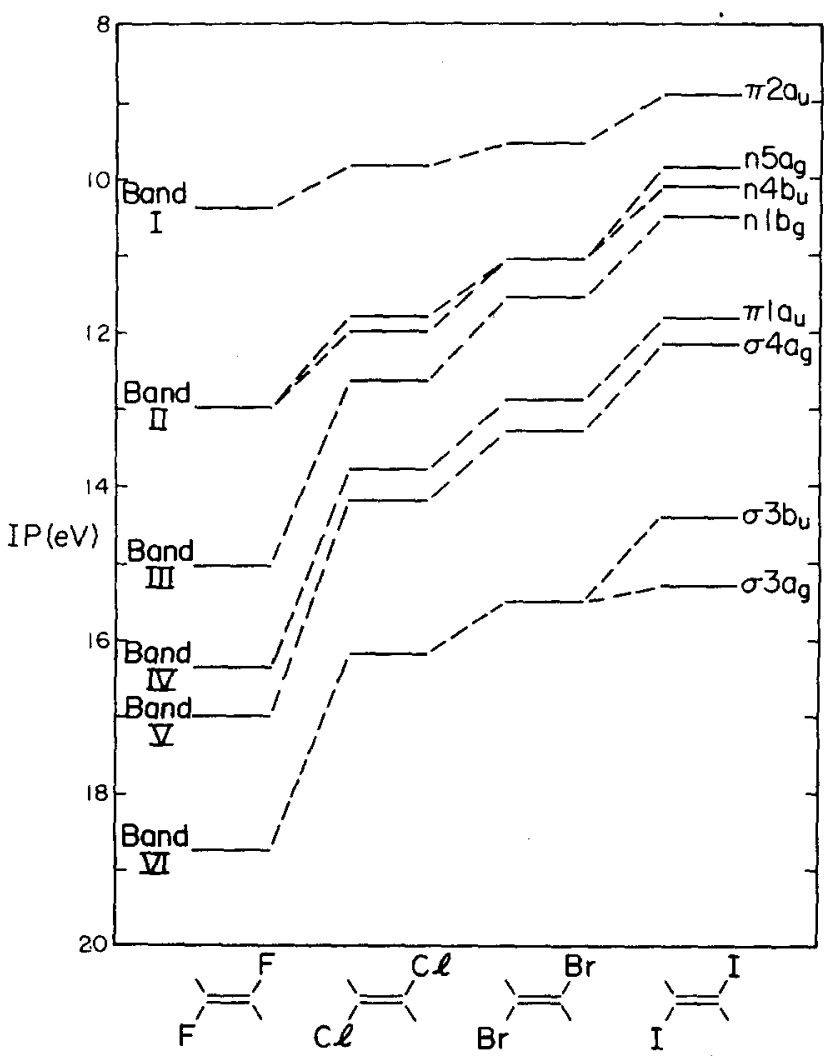

FIG. 13. Correlation of energy levels for the trans-dihalogenated ethylenes derived by photoelectron spectroscopy. The orbital energies and assignments for trans $-1,2-\mathrm{C}_{2} \mathrm{H}_{2} \mathrm{I}_{2}$ are taken from Ref. 33 and for trans $-1,2-\mathrm{C}_{2} \mathrm{H}_{2} \mathrm{Br}_{2}$ and trans $-1,2-\mathrm{C}_{2} \mathrm{H}_{2} \mathrm{Cl}_{2}$ from Ref. 31. The orbital energies of trans $-1,2-\mathrm{C}_{2} \mathrm{H}_{2} \mathrm{~F}_{2}$ are those of the present experiments: the assignments for it are derived on the basis of these correlations, the $\beta$ values, and vibrational structure in the spectra (see text). 
chloroethylene. Thus, band II in trans -1, 2-difluoroethylene is very tentatively assigned to the $4 b_{u}$ and $5 a_{g}$ orbitals; in cis-1,2-difluoroethylene, it is assigned to the $5 a_{1}$ and $4 b_{2}$ orbitals.

It is not clear why the vibrational structure for this band is so different in these two isomers. It is also surprising that band II of cis-1,2-difluoroethylene has only one main vibrational progression [the $\nu_{2}(\nu \mathrm{C}=\mathrm{C}) a_{1}$ one], if it indeed corresponds to ionization from two different orbitals. The assignment of band III in trans1,2 -difluoroethylene is to the $1 b$ fluorine nonbonding orbital and to the $1 a_{2}$ one in cis-1,2-difluoroethylene. This assignment is more certain than those for band II since band III has the expected sharpness of a nonbonding orbital, and a low value of $\beta$ in anology with fluorobenzene. ${ }^{g(x)}$ The assignments for bands IV and $V$ are based solely on the correlations with the other dihalo compounds as shown in Fig. 13 and should therefore be regarded as tentative. Finally, band VI in each compound can be assigned to two orbitals $\left(3 a_{g}\right.$ and $3 b_{u}$ in the trans and $3 a_{1}$ and $4 a_{1}$ in the cis isomer) based on the correlation diagrams and on the $\beta$ spectra. The rapid variation of $\beta$ across this band in each molecule suggests the presence of more than one underlying transition.

\section{4. $1,1-\mathrm{C}_{2} \mathrm{H}_{2} \mathrm{~F}_{2}$}

The only orbital assignment that can be made with any certainty at this time is that band I corresponds to the $2 b_{1} \pi$ orbital. In addition, from the variation of $\beta$ across band III, one may conclude that this band probably corresponds to ionization from at least two orbitals There is no resolved vibrational structure in Fig. 3, except for that of bands I and II, to help us in making assignments. Correlations between this molecule and 1,1-dichloro-, -dibromo-, and di-iodoethylene are not at all clear, possibly due to the high degree of interactions between the two halogen atoms in these molecules.

\section{5. $\mathrm{C}_{2} \mathrm{HF}_{3}$}

The only orbital assignment that can be made is that band I corresponds to ionization from the least bound $\pi$ orbital, based upon its I. P., its vibrational frequencies, and the variation of $\beta$ with the degree of fluorine substitution of Fig. 10 .

\section{6. $C_{2} F_{4}$}

Band I can be assigned to the least bound $\pi$ orbital $2 b_{3 u}$ in analogy with the other molecules and based on its I. P., its vibrational frequencies, the variation of $\beta$ with the degree of fluorine substitution of Fig. 10, and calculations. ${ }^{15(2), 17}$ There are so many transitions associated with the $\mathrm{C}-\mathrm{F}$ and nonbonding $\mathrm{F}$ orbitals that correspond to bands II through $\mathrm{V}$ that there is little hope of making these assignments at the present level of resolution.

\section{SUMMARY AND CONCLUSIONS}

The He I photoelectron spectra of the six fluorine derivatives of ethylene have been obtained at a detector angle of $54.7^{\circ}$ and compared with that of ethylene. The spectral bands which had vibrational structure were measured under high resolution $(20 \mathrm{meV})$ and the vibrational frequencies and corresponding modes were assigned. Most importantly, the asymmetry parameter $\beta$ was measured across each band of these spectra. It was noticed that there was a monotonic decrease in $\beta$ upon increasing fluorine substitution for the lowest 1. P. $\pi$ band which was interpreted as due to the resonant mixing of the fluorine lone-pair $\pi$ electrons with the $\mathrm{C}=\mathrm{C} \pi$ electrons. The I. P.'s of monofluoro-, cis-1,2-difluoro-, and trans-1,2-difluoroethylene were correlated with those in the corresponding chloro-, bromo-, and iodoethylenes published previously. All of the above data were used to make some assignments of the spectral bands of these fluoroethylenes to the corresponding orbitals.

The information available so far does not permit reliable assignments to be made for most of the ionization bands of the remaining molecules considered. Nevertheless, the photoelectron and $\beta$ spectra obtained, together with future experimental and theoretical work, may eventually lead to such assignments.

${ }^{1}$ (a) R. Morgenstern, A. Niehaus, and M. W. Ruf, Chem. Phys. Lett. 4, 635 (1970); (b) A. Niehaus and M. W. Ruf, ibid. 11, 55 (1971).

${ }^{2}$ (a) T. A. Carlson, Chem. Phys. Lett. 9, 23 (1971); (b) T. A. Carlson and C. P. Anderson, ibid. 10, 561 (1971); (c) T. A. Carlson and A. E. Jonas, J. Chem. Phys. 55, 4913 (1971); (d) T. A. Carlson, G. F. McGuire, A. E. Jonas, K. L. Cheng, C. P. Anderson, C. C. Lu, and B. P. Pullen, in Electron Spectroscopy, edited by D. A. Shirley (NorthHolland, Amsterdam, 1972), pp. 207-232.

${ }^{3}$ (a) D. C. Mason, A. Kuppermann, and D. M. Mintz, in Ref. 2(d), pp. 269-275. The values of $\beta$ presented in this reference have been superceded by those in Refs. $9(\mathrm{~d})$ and 9 (g).

${ }^{4}$ (a) T. A. Carlson and G. E. McGuire, J. Electron Spectrosc. 1, $209(1972 / 73)$; (b) T. A. Carlson and R. M. White, Discuss. Faraday Soc. 54, 285 (1972); (c) R. M. White, T. A. Carlson, and D. P. Spears, J. Electron Spectrosc. 3, 59 (1974).

${ }^{5}$ V. B. Milyaev and F. I. Vilesov, High Energy Chem. 7, 414 (1.973).

${ }^{6}$ T. Kobayashi and S. Nagakura, J. Electron Spectrose. 7, 187 (1975).

${ }^{7}$ W. H. Hancock and J. A. R. Samson, J. Electron Spectrose. 9, 211 (1976).

${ }^{8}$ F. Leng and G. Nyberg, J. Electron Spectrosc. 11, 293 (1977).

${ }^{9}$ (a) J. A. Sell and A. Kuppermann, Chem. Phys. 33, 367 (1978); (b) 33, 379 (1978); (c) J. A. Sell and A. Kuppermann, Chem. Phys. Lett. 61, 355 (1979); (d) J. A. Sell, D. M. Mintz, and A. Kuppermann, Chem. Phys. Lett. 58, 601 (1978); J. A. Sell, A. Kuppermann, and D. M. Mintz, J. Electron Spectrosc. 16, 127 (1979); (f) D. M. Mintz and A. Kuppermann, J. Chem. Phys. 69, 3953 (1978); (g) D. M. Mintz and A. Kuppermann, "Photoelectron spectroscopy of ethylene, isobutylene, 2-methyl-2-butene, and 2,3-dimethyl2-i)utene," J. Chem. Phys. (to be published); (h) D. M. Mintz and A. Kuppermann, "Variable angle photoelectron spectroscopy of allene, 1, 3-butadiene, 1,4-pentadiene, 1;5hexadiene, and 1,4-cyclohexadiene," J. Chem. Phys. (submitted).

${ }^{10}$ (a) J. Cooper and R. N. Zare, J. Chem. Phys. 48, 942 
(1968); (b) J. L. Hall and M. W. Seigel, J. Chem. Phys. 48, 943 (1968).

${ }^{11}$ (a) J. C. Tully, R. S. Berry, and B. J. Dalton, Phys. Rev. 176, 95 (1968); (b) A. D. Buckingham, B. J. Orr, and J. M. Sichel, Philos. Trans. R. Soc. London Ser. A 268, 147 (1970).

${ }^{12}$ (a) D. J. Kennedy and S. T. Manson, Phys. Rev. A 5, 227 (1972); (b) S. T. Manson, J. Electron Spectrosc. 1, 413 $(1972 / 73)$; (c) N. M. Kabachnik and I. P. Sazhina, J. Phys. B 9, 1681 (1976); (d) K. T. Taylor, ibid. 10, L699 (1977); (e) D. L. Miller, J. D. Dow, R. G. Houlgate, G. V. Marr, and J. B. West, ibid. 10, 3205 (1977); (f) W. Ong and S. T. Manson, ibid. 11, L65 (1978).

${ }^{13}$ (a) J. J. Huang and F, O. Ellison, Chem. Phys. Lett. 28, 565 (1974); (b) F. M. Chapman, Jr., and E. F. Hayes, J. Chem. Phys. 67, 2974 (1977); (c) Y. Itikawa, Chem. Phys. 28, 461 (1978); (d) 30,109 (1978).

${ }^{14}$ (a) S. Iwata and S. Nagakura, Mol. Phys. 27, 425 (1974); (b) J. W. Rabalais, T. P. Debies, J. I. Berkosky, J. J. Huang, and F. O. Ellison, J. Chem. Phys. 61, 529 (1974); (c) B. Ritchie, ibid, 60, 898 (1974); (d) 61, 3279 (1974); (e) 61, 3291 (1974); (f) 63, 1351 (1975); (g) F. Hirota, J. Electron Spectrosc. 9, 149 (1976); (h) C. Duzy and R. S. Berry, J. Chem. Phys. 64, 2421 (1976); (i) O. F. Kalman, Mol. Phys. 34, 397 (1977).

${ }^{15}$ (a) C. R. Brundle, M. B. Robin, N. A. Kuebler, and H. Basch, J. Am. Chem. Soc. 94, 1451 (1972); (b) C. R. Brundle, M. B. Robin, and N. A. Kuebler, ibid. 94, 1466 (1972). ${ }^{16} \mathrm{~S}$. Meza and U. Wahlgren, Theor. Chim. Acta 21, 323 (1971). ${ }^{17} \mathrm{~J}$. A. Pappas, J. Mol. Struct. 22, 69 (1974).

${ }^{18}$ D. C. Mason, D. M. Mintz, and A. Kuppermann, Rev. Sci. Instrum. 48, 926 (1977).

${ }^{19}$ This manometer is the MKS Baratron model 210 (MKS Instruments, Inc., Burlington, MA 01803) which has been calibrated at the factory against an air dead-weight tester. The calibration is reported to be accurate to within $0.1 \%$ over the pressure range $10^{-5}$ to 1 Torr.

${ }^{20}$ G. J. Schulz and A. V. Phelps, Rev. Sci. Instrum. 28, 1051 (1957). One commercial source of this gauge is the Granville-Phillips Co., Boulder, CO 80303.

${ }^{21}$ J. A. R. Samson, J. Opt. Soc. Am. 59, 356 (1969).

${ }^{22}$ J. A. R. Samson, Philos. Trans. R. Soc. London Ser. A 288, 141 (1970).

${ }^{23}$ D. W. Turner, C. Baker, A. D. Baker, and C. R. Brundle, Molecular Photoelectron Spectroscopy (Wiley-Interscience, London, 1970), pp. 166-168.

${ }^{24}$ A. D. Baker, C. Baker, C. R. Brundle, and D. W. Turner,
Int. J. Mass Spectrom. Ion Phys, 1, 285 (1968).

${ }^{25}$ V. D. Reinke, H. Baumgärtel, T. Cvitas, L. Klasinc, and H. Güsten, Ber. Bunsenges. Phys. Chem. 78, 1145 (1974).

${ }^{26}$ R. F. Lake and H. Thompson, Proc. R. Soc. (London) 315, 323 (1970).

${ }^{27}$ Reference 23, p. 245.

${ }^{28}$ Reference 23 , p. 247.

${ }^{29}$ D. P. Chong, F. G. Herring, and D. McWilliams, J. Chem. Soc. Faraday Trans. 2 70, 193 (1974).

${ }^{30}$ D. Chadwick, A. B. Cornford, D. C. Frost, F. G. Herring, A. Katrib, C. A. McDowell, and R. A. N. McLean, in Ref. 2(d), pp. 453-470.

${ }^{31} \mathrm{~K}$. Wittel and H. Bock, Chem. Ber. 107, 317 (1974).

${ }^{32}$ D. Chadwick, D. C. Frost, A. Katrib, C. A. McDowell, and R. A. N. McLean, Can. J. Chem. 50, 2642 (1972).

${ }^{33} \mathrm{~K}$. Wittel, H. Bock, and R. Manne, Tetrahedron 30, 651 (1974).

${ }^{34}$ D. C. Frost and J. S. Sandhu, Indian J. Chem. 9, 1105 (1971).

${ }^{35}$ N. D. Epiotis, J. Am. Chem. Soc. 95, 3087 (1973).

${ }^{36}$ J. S. Binkley and J. A. Pople, Chem. Phys. Lett. 45, 197 (1977).

${ }^{37}$ C. B. Duke, K. L. Yip, G. P. Ceasar, A. W. Potts, and D. G. Streets, J. Chem. Phys. 66, 256 (1977).

${ }^{38}$ A. Savitzky and M. Golay, Anal. Chem. 36, 1627 (1964).

${ }^{39} \mathrm{Copies}$ of the high resolution vibrational spectra for the seven molecules are available from the authors upon request.

${ }^{40} \mathrm{G}$. R. Branton, D. C. Frost, T. Makita, C. A. McDowell, and I. A. Stenhouse, Philos. Trans. R, Soc. London Ser. A 268, 77 (1970).

${ }^{4 !}$ D. C. McKean, Spectrochim. Acta Part A 31, 1167 (1975).

${ }^{42}$ D. C. Smith, J. R. Nielsen, and H. H. Claassen, J. Chem. Phys. 18, 326 (1950).

${ }^{43}$ N. C. Craig and J. Overend, J. Chem. Phys. 51, 1127 (1969).

${ }^{44}$ D. E. Mann, N. Acquista, and K. Plyler, J. Chem. Phys. 22, 1586 (1954).

${ }^{45}$ A. J. Merer and L. Schoonveld, Can. J. Phys. 47, 1731 (1969).

${ }^{46}$ R. Bralsford, P. V. Harris, and W. C. Price, Proc. R. Soc. (London) 258, 459 (1960).

${ }^{47}$ I. Fischer-Hjalmars and S. Meza, Acta Chem. Scand. 26, 2991 (1972).

${ }^{48}$ T. Hayashi and T. Nakajima, Bull. Chem. Soc. Jpn. 49, 2055 (1976).

${ }^{49} \mathrm{~T}$. Koopmans, Physics (Utrecht) 1, 104 (1933).

${ }^{50}$ D. T. Clark, J. N. Murrell, and J. M. Tedder, J. Chem. Soc. $1963,1250$. 